\title{
Hypoxia promotes growth of stem cells in dental follicle cell populations
}

\author{
Yuntao Dai, Hongzhi He, Gary E. Wise, Shaomian Yao*
}

Department of Comparative Biomedical Sciences, School of Veterinary Medicine Louisiana State University, Baton Rouge, USA. Email: shaomia@1su.edu

Received 8 April 2011; revised 15 May 2011; accepted 29 May 2011.

\begin{abstract}
Adult stem cells (ASC) have been found in many tissues and are of great therapeutic potential due to their capability of differentiation. However, ASC comprise only a small fraction of the tissues. In order to use ASC for therapeutic purposes, it is important to obtain relatively pure stem cells in large quantities. Current methods for stem cell purification are mainly based on marker-dependent cell sorting techniques, which have various technical difficulties. In this study, we have attempted to develop novel conditions to favor the growth of the dental follicle stem cells (DFSC) such that the resultant cell populations are enriched in stem cells. Specifically, a heterogeneous dental follicle cell (H-DFC) population containing stem cells and homogenous non-stem cell dental follicle cell population were cultured at $1 \%$ or $5 \%$ hypoxic conditions. Only the heterogeneous population could increase proliferation in the hypoxic condition whereas the homogenous DFC did not change their proliferation rate. In addition, when the resultant cells from the heterogonous population were subjected to differentiation, they appeared to have a higher capacity of adipogenesis and osteogenesis as compared to the controls grown in the normal atmosphere (normoxic condition). These hypoxiatreated cells also express higher levels of some stem cell markers. Together, these data suggest that stem cells are enriched by culturing the heterogeneous cell populations in a reduced $\mathrm{O}_{2}$ condition.
\end{abstract}

Keywords: Hypoxia; Dental Follicle (DF); Stem Cells; Proliferation; Gene Expression

\section{INTRODUCTION}

Stem cells in adult tissues, adult stem cells (ASC), function as replenishment or repair systems for maintaining the integrity of the given tissue. ASC are unspecialized cells that are capable of differentiating into specialized cells. Due to this characteristic, ASC have great potential in medical applications such as in tissue regeneration and tissue engineering.

The dental follicle is a loose connective tissue sac surrounding the unerupted tooth and its presence is required for the tooth to erupt $[1,2]$. Our previous studies revealed that the dental follicle plays a critical role in regulating alveolar bone resorption and bone formation for tooth eruption by regulating formation of osteoclasts and osteoblasts $[3,4]$. Stem cells have been found in the dental follicle, and dental follicle stem cells (DFSC) are capable of multilineage differentiation including osteogenesis [5]. Thus, we proposed that stem cells in the dental follicle may differentiate into osteoblasts to promote some of the bone formation needed for tooth eruption. Therefore, studies of DFSC might help understand the cell and molecular mechanisms of tooth eruption. Moreover, the mutilineage differentiation potential of the DFSC suggests that they would be valuable for tissue engineering applications.

Isolation of ASC has often relied on their plastic adherence preference. Because many non-stem cells can also adhere to and grow on plastic surfaces, the cell population isolated based on plastic adherence contains many non-stem cells. For example, adherent single cellderived colonies of progenitors isolated from bone marrow display a wide variation in cell morphology and growth potential [6]. Alternatively, stem cells may be isolated based on their colony-forming properties, a method which has been used to isolate pulp stem cells from human third molars [7] and from human exfoliated deciduous teeth [8]. However, such isolation techniques are labor-intensive, time-consuming and skill-demanding. Colonies derived from heterogeneous dental pulp cells also show diversity in cell surface markers [7], suggesting that the technique does not result in a pure population of stem cells.

Cell marker-dependent sorting techniques, including 
fluorescence-activated cell sorting (FACS) and magnetic-activated cell sorting (MACS) have been developed for isolation of stem cells from various tissues. Such techniques require the presence of stem cell specific markers, especially surface markers. The major drawback for application of the sorting techniques is the lack of clear stem cell specific markers. If no unique stem cell-specific surface marker is present, selection must be repeated with multiple markers, which greatly increases the cost while reducing the efficacy of the methods. Therefore, such techniques do not allow purifying stem cells on a large scale. In vitro expansion of the primary isolated ASC still is required to obtain a large quantity for clinical applications.

It is believed that stem cells in adult tissues function as the repair/regeneration systems to regenerate damaged or defective tissues/organs. Tissue damage can occur in several situations, most often in a disease state. For example, many diseases can cause a shortage of oxygen supply in the tissues (i.e., hypoxia), and in turn may cause tissue damage that requires the tissue stem cells to function for regeneration. Theoretically, the stem cells must remain intact in the hypoxic condition and respond to hypoxia. It has been reported that stem cells grow more rapidly and form more colonies in hypoxic conditions than they do in normal oxygen atmosphere $[9,10]$. Thus, it is our hypothesis that DFSC are more responsive to hypoxia than normal somatic cells, and hypoxia may activate the stem cells from quiescence to begin rapid growth. We further propose that this unique feature can be used to develop conditions that favor stem cell growth such that one could enrich stem cells from heterogeneous cell populations.

To that end, a homogeneous dental follicle cell (DFC) population containing only fibroblast-like cells and a heterogeneous dental follicle cell (H-DFC) population containing fibroblast-like cells and stem cells were incubated under hypoxic conditions. The effects of hypoxia on cell proliferation, differentiation and selected stem cell marker gene expression were studied. The possibility of enrichment of stem cells by growing the cells in hypoxic conditions is also discussed.

\section{MATERIALS AND METHODS}

\subsection{Cell Culture}

Dental follicles were isolated from the first mandibular molars of rat pups at postnatal days 5-7, and then trypsinized to obtain the primary dental follicle cell suspension. The primary cells were cultured in Eagle's minimum essential medium containing $10 \%$ newborn calf serum and $1 \mathrm{mM}$ sodium pyruvate [11]. Cells were passed at confluency until the desired passage to obtain a pure fibroblast-like cell population containing no stem cells [5]. This population is referred to as dental follicle cells (DFC) in this manuscript. To obtain the heterogeneous cell population containing dental follicle stem cells (DFSC) and non-stem cells, the primary cells were cultured in alpha minimal essential medium (Invitrogen) mixed with $20 \%$ fetal bovine serum (Atlanta Biologicals, Lawrenceville, GA) and also passed at confluence. The cell population obtained in this condition is heterogeneous containing stem cells and non-stem cells [9] and is referred to as heterogeneous dental follicle cells $(\mathrm{H}-$ DFC). All cultures were incubated at $37^{\circ} \mathrm{C}$ with $5 \% \mathrm{CO}_{2}$ atmosphere unless otherwise specified.

Hypoxic cultures were achieved using the MIC-101 hypoxia chamber (Billups-Rothenberg, Inc.). The cells were seeded in flasks or plates and then the flasks or plates were placed in the chambers. The chambers were then filled with a gas mixture containing the designated concentrations of $\mathrm{O}_{2}$ and $5 \% \mathrm{CO}_{2}$, balanced with $\mathrm{N}_{2}$. Next, the chambers were moved into the $37^{\circ} \mathrm{C}$ incubator. $\mathrm{O}_{2}$ concentrations were monitored daily with the $\mathrm{O}_{2}$ meter inside the chambers. Cell culture media were changed every other day.

\subsection{Gene Expression Study}

Gene expression was determined using real-time RTPCR, Western blotting or cytochemistry staining after 7 days of incubation with the designated treatments. For real-time RT-PCR, total RNA was extracted with RNeasy Mini Kit (Qiagen); RNA concentration and quality was measured with a Nanodrop 8000 spectrophotometer (Thermo scientific). RNA ( $2 \mu \mathrm{g}$ per sample) was reverse transcribed into $20 \mu \mathrm{l}$ cDNA with random primers and MLV reverse transcriptase. Next, SYBR green real-time PCR was conducted with $0.5 \mu \mathrm{l}$ cDNA of each sample using gene specific primers to determine the expression of $N t 5 e$ (forward: 5'ACTCCACCAAG TGCCTCAAC3'; reverse 5' GTCCTTCCACACCGTTA TCAA3') and Proml (forward: 5'GGGAGC GAGATG TTACTTTGA G3'; reverse: 5'CAGC AGGACACT GAATAC GAGA3').

For Western blotting, total protein was extracted from the cells with the Cytobuster Protein Extraction Reagent (Novagen), and quantified with the BCA Protein Assay Kit (Pierce). An equal amount of total protein $(20 \mu \mathrm{g})$ from each sample was loaded onto a SDS-polyacrylamide gel for electrophoresis. The proteins were transferred to a membrane after the electrophoresis. The membrane was hybridized with $12.5 \mu \mathrm{g} / \mathrm{ml}$ rabbit polyclonal anti-Prom1 or anti-Nt5e antibodies (Abcam) after treatment of the membrane with $5 \%$ dry milk to block nonspecific binding. The membrane was washed with PBS-T for 6 times to remove unbound antibodies, and then hybridized with anti-rabbit secondary antibody conjugated to horseradish peroxidase. The protein signal 
was detected by an enhanced chemiluminescence kit (Santa Cruz) with a Kodak X-ray film.

For alkaline phosphatase (ALP) staining, H-DFC and DFC were seeded in 6 well plates and cultured at the designated hypoxic condition. After 7 days of incubation, cells were stained for cell membrane ALP using Stem$\mathrm{TAG}^{\mathrm{TM}}$ Alkaline Phosphatase Staining Kit (Cell Biolabs, Inc.) according to the manufacturer's protocol. Cells maintained in the normal culture condition (i.e., normoxia) were used as the controls.

\subsection{Determination of Cell Growth}

Because the Alamar blue assay is a non-toxic method that allows us to continuously monitor the cell growth during a period of time in culture, this method was used to monitor cell growth. To do that, DFC and H-DFC were seeded in the wells of 6-well plates. Alamar blue assays were conducted at days 1, 3, 5, and 7 of culture. At the time of assay, the culture medium was removed from the wells and assay medium containing 10\% Alamar blue was added to each well. After 2 hours of incubation, $100 \mu$ assay medium was pipetted into a well of the 96-well plate in triplicate for each sample. Fresh cell culture medium and fresh assay medium not subjected to cell incubation were used as the controls. The 96-well plate was placed into a Bio-Rad microplate reader (model 550) and OD values were obtained. Alamar blue reduction was calculated according to the manufacturer's formula.

\subsection{Cell Cycle Analysis}

Cell cycle analysis was conducted to determine if the H-DFC cultured under hypoxic conditions divided more than under normoxic conditions. Approximately 3 $\times 10^{5}$ cells from each of the treatments were seeded per $75 \mathrm{~cm}^{2}$ T-flask in $20 \mathrm{ml}$ medium with a change of medium at 2- day intervals. The cells were collected on day 4 of culture for cell cycle analysis using flow cytometry. Briefly, the cells were pre-treated with cold methanol followed by treatment with Rnase A and Propidium iodide. DNA content of the cells was determined using a Becton Dickinson FACScan flow cytometer (San Jose). Percentages of cells in G0/G1, S, and $\mathrm{G} 2 / \mathrm{M}$ phases of the cell cycle were determined for each sample.

\subsection{Cell Differentiation}

To determine the effect of hypoxia on differentiation of resultant H-DFC, H-DFC were collected after 7 days of hypoxic incubation, and seeded onto 6-well plates with an concentration of $2 \times 10^{4}$ cells per well with $3 \mathrm{ml}$ differentiation medium for induction of either adipogenesis or osteogenesis as described in our previous publication [5]. The plates were incubated at $37^{\circ} \mathrm{C}$ and $5 \% \mathrm{CO}_{2}$ for
2 weeks. Next, the cells were stained with Alizarin Red Solution (Sigma-Aldrich) for assessing osteogenesis, and with Oil Red O (Sigma-Aldrich) for adipogenesis.

\subsection{Statistical Analysis}

All experiments were repeated three times. Treatment effects were compared by analysis of variance with SAS version 9.1, and means were separated using least significant difference (LSD) at $P \leq 0.05$.

\section{RESULTS}

\subsection{Increase of cell Growth by Hypoxia}

After 7 days of culture, H-DFC showed higher cell density in both $1 \%$ and $5 \%$ hypoxia treatments as compared to the normoxia control whereas similar cell density was seen for DFC in all treatments (Figure 1(a)). We conducted an Alamar blue assay to monitor cell proliferation during 7 days of culture. H-DFC had an overall higher Alamar blue reduction than DFC in all treatments after 5 and 7 days of incubation (Figure 1(b)), indicating that $\mathrm{H}-\mathrm{DFC}$ proliferated more rapidly than DFC. Alamar blue reduction (i.e., cell proliferation) was significantly higher when $\mathrm{H}$-DFC were incubated under $1 \%$ and $5 \% \mathrm{O}_{2}$ hypoxic conditions than under normoxic conditions after 5 and 7 days of incubation. The maximum Alamar blue reduction was seen at $5 \% \mathrm{O}_{2}$, indicating that $\mathrm{H}$-DFC grew most rapidly at $5 \% \mathrm{O}_{2}$ (Figure $1(\mathrm{~b})$ ). In contrast to $\mathrm{H}-\mathrm{DFC}$, culture of DFC in different conditions (hypoxic or normoxic) resulted in a similar Alamar blue reduction within each given day of incubation, suggesting that DFC did not respond to hypoxia treatment.

To further study cell division, cell cycle analysis was carried out by flow cytometry. Cell cycle analysis of $\mathrm{H}-\mathrm{DFC}$ showed that the average percentage of cells in S-phase was $19.11 \%$ in $1 \% \mathrm{O}_{2}, 22.37 \%$ in $5 \% \mathrm{O}_{2}$ and $16.07 \%$ in the control (normoxia) at day 4 of culture (Figure 1(c)). The difference was statistically significant $(P \leq 0.05)$, suggesting that a greater number of cells was in the dividing stage under hypoxic conditions as compared to under the normoxic condition.

\subsection{Increase of Differentiation Capability after Hypoxic Treatment}

After 1 week of culture in hypoxia, the resultant H-DFC populations were induced for adipogenesis and osteogenesis. Adipogenesis and osteogenesis were determined by Oil Red O staining or Alizarin Red staining, respectively. This study showed that the cell populations grown in $1 \%$ and $5 \%$ hypoxia had greater osteogenesis and adipogenesis than did the control cells grown in nor- 

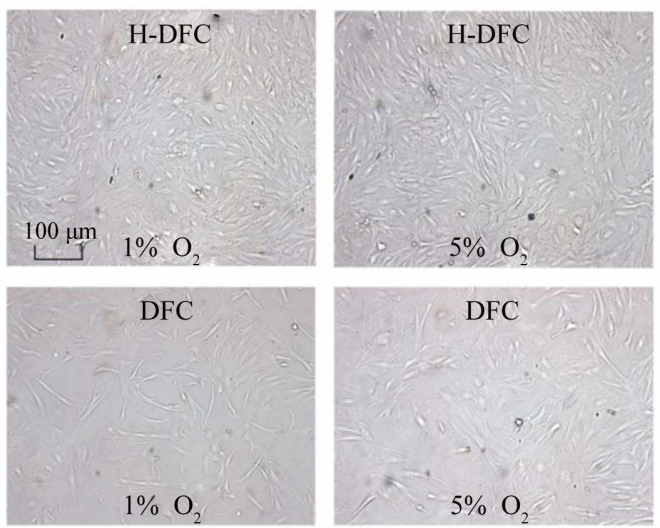

(a)
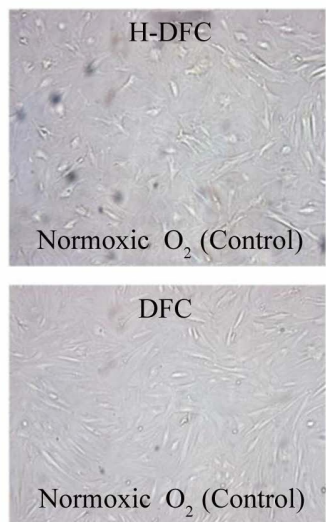

Normoxic $\mathrm{O}_{2}$ (Control)
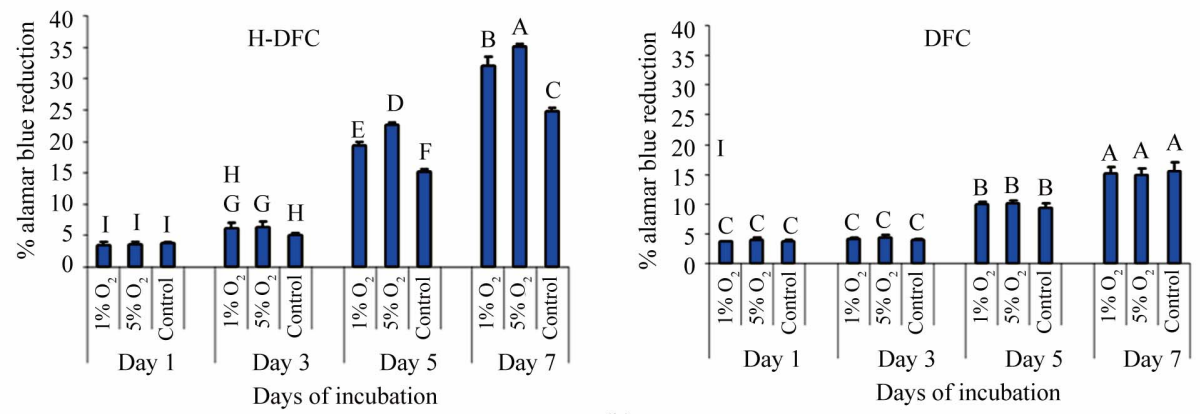

(b)

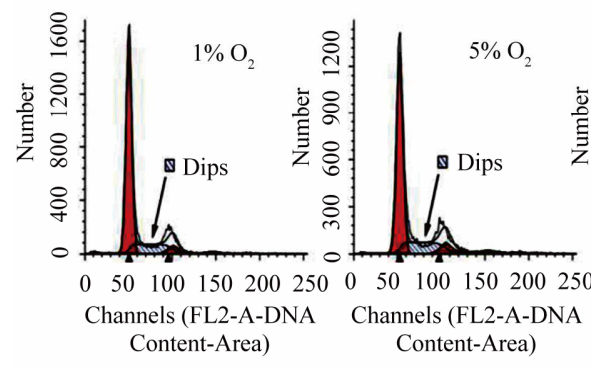

(c)

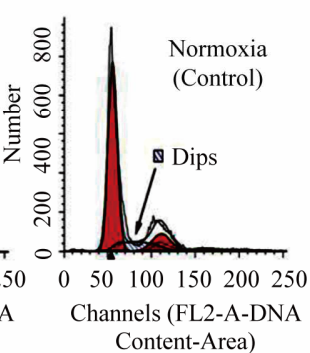

Content-Area)

Days of incubation

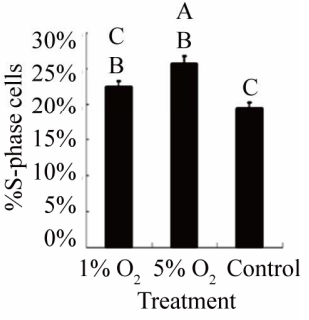

(d)

Figure 1. Effects of hypoxia on the culture of H-DFC and DFC. Note that only H-DFC responded to the hypoxia as shown by the cell density change after a 1 week of incubation (a); and by Alamar blue cell proliferation assay during 1 week period of incubation (b). Cell cycle analysis was conducted by flow cytometry showing that hypoxia treatment resulted in a significantly higher percentage of cells in S-phase (c, d). Bars labeled with different letters indicate a statistically significant difference at $P \leq 0.05$.

moxia (Figure 2). Maximum staining was seen in the cells that had been subjected to $5 \%$ hypoxic incubation (Figure 2).

\subsection{Enhancement of Stem Cell Marker Gene Expression after Hypoxia Treatment}

Real-time RT-PCR analysis showed that expression of stem cell marker genes $\mathrm{Nt5e}$ and Proml were significantly enhanced in H-DFC incubated in hypoxic treatments $\left(1 \%\right.$ and $\left.5 \% \mathrm{O}_{2}\right)$ as compared to the controls maintained under normoxic condition (Figure 3). Cells from $5 \% \mathrm{O}_{2}$ treatment had the highest expression of
$N t 5 e$, which was also significantly higher than $1 \% \mathrm{O}_{2}$ treatment and controls (Figure 3(a)). For Prom1, although expression was higher in both $1 \%$ and $5 \% \mathrm{O}_{2}$ hypoxia treatments than in the controls, no difference was detected between $5 \%$ and $1 \% \mathrm{O}_{2}$ treatments (Figure 3(b)).

Western blotting determined that H-DFC from hypoxia treatment produced a greater amount of Prom 1 and $\mathrm{Nt5e}$ proteins than did the controls. Thus, this result suggests that the mRNA level determined by the real-time RT-PCR corresponds to the protein expression (Figure 3(c)). 


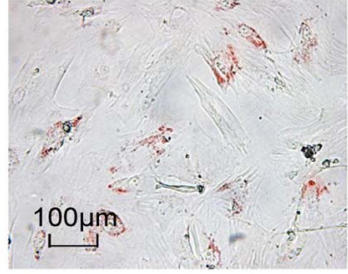

$1 \% \mathrm{O}_{2}$

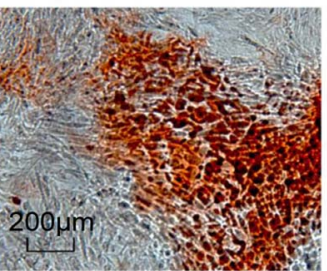

$1 \% \mathrm{O}_{2}$

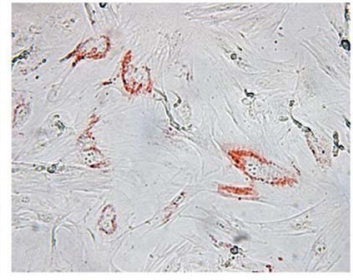

$5 \% \mathrm{O}_{2}$

(a)

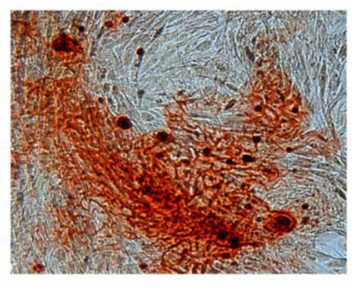

$5 \% \mathrm{O}_{2}$

(b)

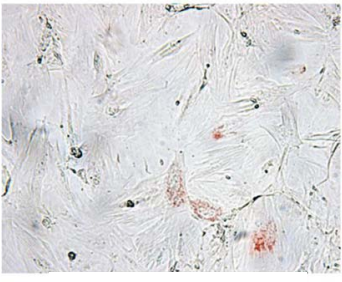

Normoxic $\mathrm{O}_{2}$ (Control)

Figure 2. Enhancement of differentiation capability of H-DFC after hypoxia treatments. Increased Oil Red O staining was seen in cells subjected to hypoxic incubation as compared to the normoxic $\mathrm{O}_{2}$ (control), indicating increased adipogenesis in hypoxia-treated cells (a); Osteogenesis was determined by Alizarin Red staining, and increased staining was seen from hypoxic treated H-DFC cells as compared to the normoxic control (b). Note that the greatest adipogenesis and osteogenesis were seen in cells resulting from $5 \% \mathrm{O}_{2}$ treatment.

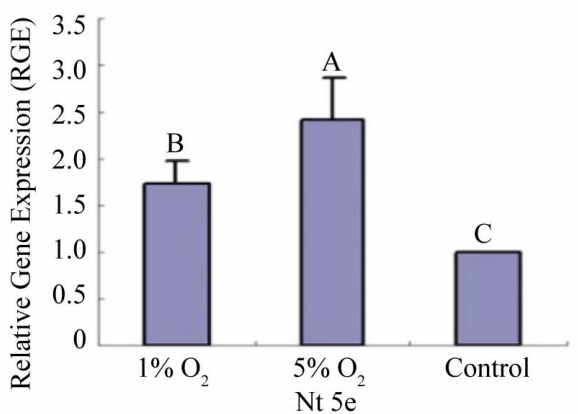

(a)

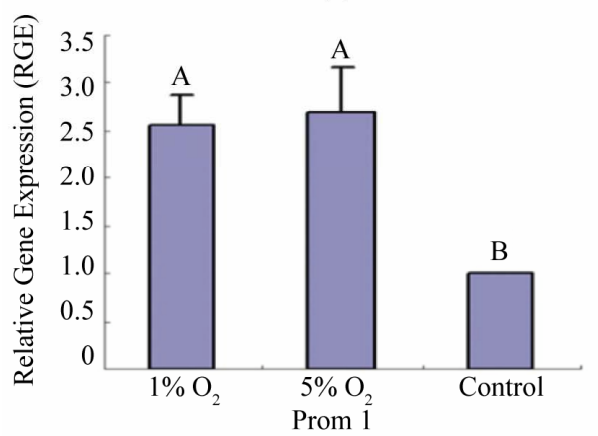

(b)

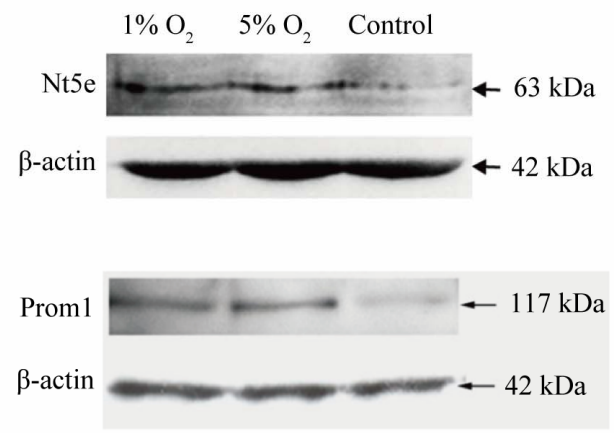

(c)

Figure 3. Enhanced expression of Nt5e and Proml genes after hypoxic treatment as determined by real-time RT-PCR $(a, b)$ and Western blotting $(c)$. Note that increased expression of both genes was statistically significant in hypoxic treatments as compared to the normoxic control. Bars with different letters indicate significant differences at $P \leq 0.05$. Western blot results indicated that the transcription and translation of the marker genes are consistent $(\mathrm{a}, \mathrm{b}, \mathrm{c})$. 


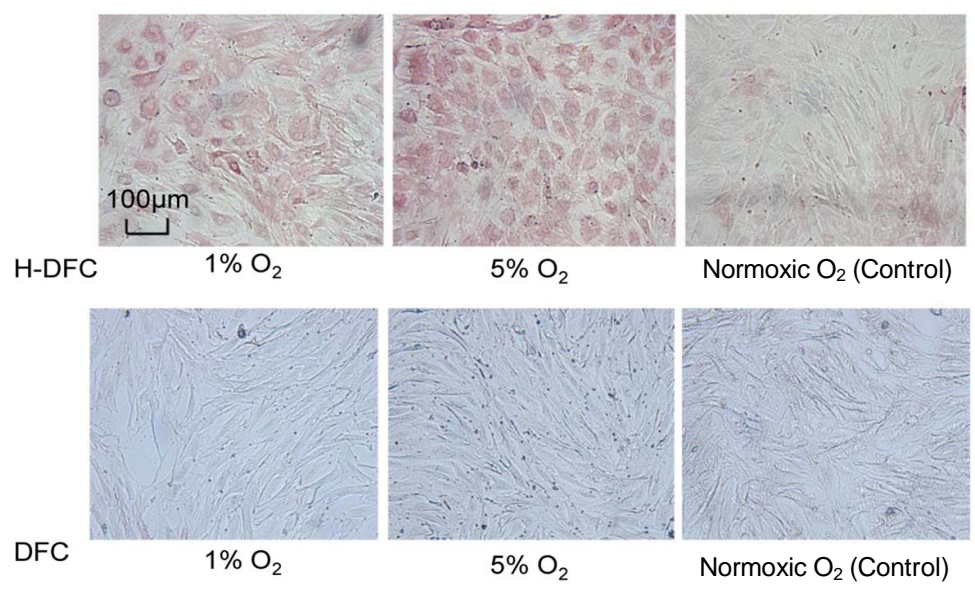

Figure 4. Alkaline phosphatase (ALP) staining of H-DFC and DFC to detect membrane ALP after 4 days of incubation in hypoxia. ALP staining was only seen in the H-DFC population, and not in the DFC. Note that the numbers of cells stained positive for ALP dramatically increased in the H-DFC population after hypoxic incubation as compared to the H-DFC population not subjected to hypoxia (Normoxic $\mathrm{O}_{2}$ ).

H-DFC from different treatments were also stained for membrane ALP. The results showed that a greater number of cells stained for membrane ALP in hypoxiatreated cells as compared to the cells grown under normoxic condition (Figure 4). Comparing 1\% and 5\% $\mathrm{O}_{2}$ treatment, $5 \% \mathrm{O}_{2}$ treatment resulted in more cells showing strong ALP staining than $1 \% \mathrm{O}_{2}$ treatment. In contrast, no ALP staining was observed in the DFC (nonstem cells) incubated either under hypoxic or normoxic conditions (Figure 4).

\section{DISCUSSION}

Sufficient quantities of stem cells must be obtained before they can be used for clinical treatments. It is difficult to collect large numbers of adult stem cells from primary isolation because 1) stem cells are rare in adult tissues; 2) there are limitations of current methods for stem cell purification; and 3) large tissue samples are often unavailable. The last is especially true for isolating stem cells from tissues of small size, such as the dental follicle. Thus, in vitro expansion of primary isolated stem cells is required in most cases in order to have sufficient numbers of cells for clinical treatments.

We have previously shown that stem cells can be isolated from the dental follicle by growing cells in $\alpha$-MEM plus $20 \%$ FBS medium, and the cell population is heterogeneous (i.e., H-DFC) with some stem cells and a majority of non-stem cells [5]. However, if the cells are grown and passaged in MEM plus $10 \%$ NCS, a homogenous fibroblast-like cell population containing no stem cells can be achieved after several passages $[5,11]$.
In this study, we show that the H-DFC population showed accelerated proliferation under hypoxic conditions as compared to the control incubated under normoxic conditions (Figure 1). In contrast, the homogenous DFC population (containing only non-stem cells) did not show notable change of its proliferation rate under either hypoxic or normoxic conditions; i.e., the DFC did not respond to hypoxic treatment. Because both populations originate from the same tissue source (the dental follicle), and because the non-stem cell homogeneous population does not increase growth rate in response to hypoxia, the increase of proliferation rate in response to hypoxia seen in the heterogeneous population may be due to more rapid growth of the stem cells than of the non-stem cells in the population.

If only the stem cells increase their proliferation such that their growth rate exceeds the growth of non-stem cells under hypoxic conditions, the ratio of stem cells to non-stem cells in the heterogeneous population should shift in favor of stem cells. To test this, the H-DFC populations were collected after 1 week of incubation under hypoxia and normoxia (control). The resultant cells were evaluated for stem cell properties of differentiation and marker gene expression. Enhancement of adipogenesis and osteogenesis was observed in the cell populations with $1 \% \mathrm{O}_{2}$ and $5 \% \mathrm{O}_{2}$ incubation (Figure 2), as compared to the control. The expression of several stem cell markers including Nt5e, Prom 1 and ALP also were increased in hypoxia-treated cells (Figures 3 and 4).

ALP, Prom 1 and Nt5e have been shown to be specific markers for certain stem cells. Specifically, expression 
of cell surface ALP is associated with undifferentiated pluripotent stem cells such as undifferentiated human pluripotent stem cells [12]. Induced pluripotent stem cells also show strong ALP activity $[13,14]$. In this study, $\mathrm{H}-\mathrm{DFC}$ resulting from 1 week hypoxia incubation show increased activity of membrane ALP.

Prom1 (also known as CD133) is well-known as a stem cell marker used for identification and isolation of adult stem cells. Prom1 was originally classified as a marker of primitive hematopoietic and neural stem cells in humans and mice $[15,16]$. Cells forming neurosphere or neural stem cells capable of multilineage differentiation were isolated based on Prom 1 expression [17-19]. Prom1 expression in adult stem cells has been shown to help in maintaining the stem cell properties by suppressing their differentiation [20].

$\mathrm{Nt5e}$ (also known as CD73) has been reported to be present on mesenchymal stem cells [21]. Expression of this gene is enhanced by hypoxia in endothelial cells $[22,23]$. In this study, there was an increased expression of $N t 5 e$ after hypoxia treatment of the H-DFC population, suggesting that hypoxia may also enhance the expression of $N t 5 e$ in stem cells. It has been proposed that increased expression of Nt5e may have protective roles during hypoxia [22,24].

Based on the evidence of increased differentiation capabilities (Figure 2) and marker gene expression after hypoxia treatment of H-DFC (Figures 3 and 4), it can be suggested that the ratio of stem cells to non-stem cells in the H-DFC populations subjected to hypoxia was increased; i.e., greater numbers of stem cells were achieved by incubating H-DFC in hypoxia than in normoxia. This is likely due to the differential growth of the stem cells and non-stem cells in response to hypoxia. In particular, stem cells significantly accelerate their proliferation rate in response to hypoxia whereas the non-stem cells (DFC) do not notably increase their proliferation rate in hypoxia (Figure 1).

Hypoxia can be a medical condition in which tissues do not receive sufficient $\mathrm{O}_{2}$ supply. Cells in a hypoxic condition undergo anaerobic metabolism, in which they accumulate lactic acid [25]. Another effect of hypoxia is stimulation of inflammation [26,27] with cell death and tissue damage occurring under severe and prolonged hypoxia. Under such conditions, the stem cells residing in the tissue may function to regenerate the damaged tissue. Because the stem cells exist in a quiescent state under normal physiological conditions and become active only when needed [28], tissue damage or the hostile conditions causing the damage may serve as signals to activate the stem cells to proliferate. This would explain why the DFSC in a H-DFC population increase their growth under hypoxic conditions (Figure 1). Increased proliferation is also seen when mesenchymal stem cells are subjected to hypoxic conditions [9].

In conclusion, incubation of heterogeneous dental follicle cell populations containing stem cells and non-stem cells in hypoxia can result in a greater increase of stem cell numbers in the population than incubation of the cells in normoxia. In particular, a $5 \% \mathrm{O}_{2}$ concentration may be optimal for growth of dental follicle stem cells (DFSC). This differential growth of the stem cells and non-stem cells in response to hypoxia may be exploited for enrichment of dental tissue stem cells from heterogeneous cell populations.

\section{ACKNOWLEDGEMENTS}

We thank Ms. Marilyn Dietrich for help with the flow cytometry study. This research was supported by NIH grant 1R03DE018998 to S. Yao.

\section{REFERENCES}

[1] Cahill, D.R. and Marks Jr, S.C. (1980) Tooth eruption: evidence for the central role of the dental follicle. Journal of Oral Pathology, 9, 189-200. doi:10.1111/j.1600-0714.1980.tb00377.x

[2] Marks Jr, S.C. and Cahill, D.R. (1984) Experimental study in the dog of the non-active role of the tooth in the eruptive process. Archives of Oral Biology, 29, 311-322. doi:10.1016/0003-9969(84)90105-5

[3] Wise, G.E. and King, G.J. (2008) Mechanisms of tooth eruption and orthodontic tooth movement. Journal of Dental Research, 87, 414-434. doi: $10.1177 / 154405910808700509$

[4] Wise, G.E. (2009) Cellular and molecular basis of tooth eruption. Orthodontics and Craniofacial Research, 12, 67-73. doi:10.1111/j.1601-6343.2009.01439.x

[5] Yao, S., Pan, F., Prpic, V., et al. (2008) Differentiation of stem cells in the dental follicle. Journal of Dental Research, 87, 767-771.

doi: $10.1177 / 154405910808700801$

[6] Bianco, P. and Gehron Robey, P. (2000) Marrow stromal stem cells. Journal of Clinical Investigation, 105, 16631668. doi:10.1172/JCI10413

[7] Gronthos, S., Mankani, M., Brahim, J., et al. (2000) Postnatal human dental pulp stem cells (DPSCs) in vitro and in vivo. Proceedings of the National Academy of Sciences, 97, 13625-13630.

doi:10.1073/pnas.240309797

[8] Miura, M., Gronthos, S., Zhao, M., et al. (2003) SHED: Stem cells from human exfoliated deciduous teeth. Proceedings of the National Academy of Sciences, 100, 5807-5812. doi:10.1073/pnas.0937635100

[9] Lennon, D.P., Edmison, J.M. and Capalan, A.I. (2001) Cultivation of rat marrow-derived mesenchymal stem cells in reduced oxygen tension: effects on in vitro and in vivo osteochondrogenesis. Journal of Cell Physiology, 187, 345-355. doi:10.1002/jep.1081

[10] Yoshida, Y., Takahashi, K., Okita, K. et al. (2009) Hypoxia enhances the generation of induced pluripotent stem cells. Cell Stem Cell, 5, 237-241. 
doi:10.1016/j.stem.2009.08.001

[11] Wise, G.E., Lin, F. and Fan, W. (1992) Culture and characterization of dental follicle cells from rat molars. Cell Tissue Research, 267, 483-492. doi:10.1007/BF00319370

[12] O'Connor, M.D., Kardel, M.D., Iosfina, I., et al. (2008) Alkaline phosphatase-positive colony formation is a sensitive,specific, and quantitative indicator of undifferentiated human embryonic stem cells. Stem Cells, 26, 11091116. doi:10.1634/stemcells.2007-0801

[13] Yu, J., Vodyanik, M.A., Smuga-Otto, K., et al. (2007) Induced pluripotent stem cell lines derived from human somatic cells. Science, 318, 1917-1920. doi:10.1126/science. 1151526

[14] Aasen, T., Raya, A., Barrero, M.J., et al. (2008) Efficient and rapid generation of induced pluripotent stem cells from human keratinocytes. Nature Biotechnology, 26, 1276-1284. doi:10.1038/nbt.1503

[15] Yin, A.H., Miraglia, S., Zanjani, E.D., et al. (1997) AC133, a novel marker for human hematopoietic stem and progenitor cells. Blood, 90, 5002-5012.

[16] Mizrak, D., Brittan, M. and Alison, M.R. (2008) CD133: molecule of the moment. Journal of Pathology, 214, 3-9. doi: $10.1002 /$ path. 2283

[17] Uchida, N., Buck, D.W., He, D., et al. (2000) Direct isolation of human central nervous system stem cells. Proceedings of the National Academy of Sciences, 97, 14720-14725. doi:10.1073/pnas.97.26.14720

[18] Lee, A., Kessler, J.D., Read, T.A., et al. (2005) Isolation of neural stem cells from the postnatal cerebellum. Nature Neuroscience, 8, 723-729. doi:10.1038/nn1473

[19] Corti, S., Nizzardo, M., Nardini, M., et al. (2007) Isolation and characterization of murine neural stem/progenitor cells based on Prominin-1 expression. Experimental Neurology, 205, 547-562. doi:10.1016/j.expneurol.2007.03.021

[20] Chen, C.H., Dixon, R.A., Ke, L.Y., et al. (2009) Vascular progenitor cells in diabetes mellitus: roles of WNT signaling and negatively charged low-density lipoprotein.
Circulation Research, 104, 1038-1040. doi:10.1161/CIRCRESAHA.109.198051

[21] Barry, F., Boynton, R., Murphy, M., et al. (2001) The SH-3 and $\mathrm{SH}-4$ antibodies recognize distinct epitopes on CD73 from human mesenchymal stem cells. Biochemical and Biophysical Research Communications. 289, 519-524. doi:10.1006/bbrc.2001.6013

[22] Synnestvedt, K., Furuta, G.T., Comerford, K.M., et al. (2002) Ecto-5'-nucleotidase (CD73) regulation by hypoxia-inducible factor-1 mediates permeability changes in intestinal epithelia. Journal of Clinical Investigation, 110, 993-1002.

[23] Ledoux, S., Runembert, I., Koumanov, K., et al. (2003) Hypoxia enhances Ecto-5'-Nucleotidase activity and cell surface expression in endothelial cells: role of membrane lipids. Circulation Research, 92, 848-855. doi:10.1161/01.RES.0000069022.95401.FE

[24] Friedman, G.B., Taylor, C.T., Parkos, C.A., et al. (1998) Epithelial permeability induced by neutrophil transmigration is potentiated by hypoxia: role of intracellular cAMP. Journal of Cell Physiology, 176, 76-84. doi:10.1002/(SICI)1097-4652(199807)176:1<76::AID-J CP9>3.0.CO;2-5

[25] Luft, F.C. (2001) Lactic acidosis update for critical care clinicians. Journal of the American Society of Nephrology, 12, S15-19.

[26] Ye, J., Gao, Z., Yin, J., et al. (2007) Hypoxia is a potential risk factor for chronic inflammation and adiponectin reduction in adipose tissue of ob/ob and dietary obese mice. American Journal of Physiology-Endocrinology and Metabolism, 293, E1118-1128. doi:10.1152/ajpendo.00435.2007

[27] Gonzalez, N.C. and Wood, J.G. (2010) Alveolar hypoxiainduced systemic inflammation: what low $\mathrm{PO}(2)$ does and does not do. Advances in Experimental Medicine and Biology, 662, 27-32. doi:10.1007/978-1-4419-1241-1_3

[28] Hill, M., Wernig, A. and Goldspink, G. (2003) Muscle satellite (stem) cell activation during local tissue injury and repair. Journal of Anatomy, 203, 89-99. doi:10.1046/j.1469-7580.2003.00195.x 